\title{
Computer Simulation as Evaluation Tool of Information Systems: Identifying Quality Factors of Simulation Modeling
}

\author{
Marvin Auf der Landwehr \\ Faculty IV - Business and Informatics \\ Hochschule Hannover \\ Hannover, Germany \\ marvin.auf-der-landwehr@hs- \\ hannover.de
}

\author{
Maik Trott \\ Faculty IV - Business and Informatics \\ Hochschule Hannover \\ Hannover, Germany \\ maik-trott@hs-hannover.de
}

\author{
Chirstoph von Viebahn \\ Faculty IV - Business and Informatics \\ Hochschule Hannover \\ Hannover, Germany \\ christoph-von.viebahn@hs-hannover.de
}

\begin{abstract}
With an increasing complexity and scale, sufficient evaluation of Information Systems (IS) becomes a challenging and difficult task. Simulation modeling has proven as suitable and efficient methodology for evaluating IS and IS artifacts, presupposed it meets certain quality demands. However, existing research on simulation modeling quality solely focuses on quality in terms of accuracy and credibility, disregarding the role of additional quality aspects. Therefore, this paper proposes two design artifacts in order to ensure a holistic quality view on simulation quality. First, associated literature is reviewed in order to extract relevant quality factors in the context of simulation modeling, which can be used to evaluate the overall quality of a simulated solution before, during or after a given project. Secondly, the deduced quality factors are integrated in a quality assessment framework to provide structural guidance on the quality assessment procedure for simulation. In line with a Design Science Research (DSR) approach, we demonstrate the eligibility of both design artifacts by means of prototyping as well as an example case. Moreover, the assessment framework is evaluated and iteratively adjusted with the help of expert feedback.
\end{abstract}

Keywords-Simulation Modeling, Design Science, Quality, Framework, Evaluation

\section{INTRODUCTION}

Modern organizations rely on a wide variety of IS to achieve operational excellence and establish efficient processes for delivering their products and services. In order to maintain high quality-standards, avoid malfunctions, ensure a seamless IS integration and facilitate the communication between IS, these systems are generally designed and developed in accordance with rigorous guidelines and predetermined process structures [1]. An integral constituent of the IS design and development process is the evaluation and validation of data, models, systems, integration design and business processes [2]. In the context of IS, evaluation can be classified into two fragments. While formative evaluation aims at producing information that is utilized within an iterative development process to improve the artifact under development and consequently serves the needs of the respective developer, summative evaluation focuses on the post-development and provides insights into the effectiveness of an artifact to relevant decision makers [1]. Depending on the given scenario and environment, different evaluation tools and methodologies can be applied to indemnify the orderly functionality of an IS. When it comes to the evaluation of systems that are too complex or inept to be set-up, executed or tested in a real-life scenario, simulation modeling has evolved as suitable, cost-efficient and reliable method to evaluate various IS design artifacts and systems [3]. Simulation models are a representation of a system employed to simulate and envisage the behavior of the system and a given set of conditions [4]. Concerning IS evaluation, simulation modeling allows to measure and assess the effectiveness of an IT artifact within a virtual space, which consequently results in reduced risks compared to assessment approaches and tests in reality. Accordingly, simulation modeling is widely used as evaluation instrument for IS artifacts within the domain of Business Informatics and employed in manifold research projects $[5,6]$. However, despite of the proven appropriateness of simulation modeling as evaluation instrument in IS, the impact of the individual quality of the overall simulation design remains inexplicit.

Quality has long been recognized as major influencing factor for the success of the overall IS design and development process [8], whereby a successful IS development program can be defined as a "process [that] leads to a high quality IS product whose use has a positive impact on the organization" [9]. Several studies and research projects have investigated the quality of models, systems, data, integration design and business processes, while insights into quality factors influencing the quality of simulation models as IS and the eligibility of simulation models as evaluation tool have not yet been provided. Improving the usefulness and effectiveness of the simulation modeling approach necessitates understanding and formalizing the factors that affect the solution's quality. In turn, the simulation methodology will be able to improve the overall organizational performance by ensuring effective operations of the IS or a given artifact.

In this paper, we address the need for a holistic view on simulation modeling quality as part of an integrated evaluation process. In order to provide guidance, both on the designcentric as well as the problem-centric component of quality assessment, we have defined two objectives for this paper:

1. The identification of quality factors that affect the design and development stages of simulation modeling within the context of IS evaluation.

2. The development of a comprehensive quality assessment framework, allowing to systematically define and evaluate the overall quality of a simulation model based on the priory defined quality factors.

To meet the objectives presented above, we apply the principles of DSR [10] to develop two design artifacts: (i) the 
quality factors on simulation modeling, which are collected and compiled by means of analyzing related literature as well as corresponding studies and (ii) the simulation quality framework, a conceptual model designed to guide the general quality assessment process and create formal representations of the quality factors. Our main goal is to support complex IS evaluation processes by establishing formal factors and structures ensuring quality standards in simulation modeling procedures and routines. While the factors presented in this contribution solely focus on the quality of simulation models and therefore tend to support the evaluation quality, other quality attributes (e.g. integration quality, business process quality) need to be considered in the overall IS design process to provide a complete quality view on a solution.

In this paper, we first provide a synopsis on related literature concerning the quality assessment of IS and simulation modelling as IS evaluation tool (Section II). Subsequently, we outline the methodology employed for our research and describe the overall research design (Section III), before we elaborate on the findings of our systematic literature review, namely the quality factors for simulation modeling (Section IV). Thereafter, we describe the proposed quality assessment framework and demonstrate its usefulness in the context of evaluation quality assurance by employing it in an example case (Section V). Finally, we conclude with our main findings and provide a discussion on implications as well as future research possibilities (Section VI).

\section{RELATED WORK}

Quality is an integral part of IS research and has been addressed in numerous studies. Since our study aims at establishing quality factors for simulation modeling as IS evaluation tool, we first provide an overview about literature addressing quality in IS and subsequently review existing evaluation concepts in IS research and simulation modelling.

\section{A. Quality in IS}

Generally, quality in IS relates to design and development facets, the result of an IS development process as well as the quality of IS products [7]. In scientific research, IS quality is often divided into two fragments, data quality $[11,12,13]$, which describes the quality of informational content in databases or a data warehouse and information system quality $[14,15]$, which relates to the quality of a particular IS product or artifact. Hence, IS quality can be characterized as a multidimensional system with different meanings according to a given context and perspective [16]. In this regard, several frameworks and models focusing on different elements such as the conceptual model of the IS, the quality of the modeling process, the definition of qualities or the fulfillment of specific quality levels are available to address quality concerns.

The data model quality evaluation framework [11] takes into account stakeholder, quality issue, quality factor, quality metric, improvement strategy as well as quality review and is concerned with assessing the overall quality of a data model by means of eight quality factors, namely completeness, integrity, flexibility, understandability, correctness, simplicity, integration and implementability. Generally, data quality is assessed by means of analyzing the data sources or the respective structure of the data. An overview about data quality attributes from quality management methodologies can be found in [19]. According to the SIQ framework, which represents a quality management framework [17], quality can be divided into three categories. Syntactic quality refers to the degree of conformance of a model instance to a priory defined syntax, whereby semantic quality evaluates the capability of an instance to adequately represent the object under consideration. Ultimately, pragmatic quality describes the perception of the model by its users.

When it comes to the development of an IS (artifact), aspired qualities are often described as non-functional requirements (NFRs), which delineate a set of constraints and conditions for an IS. The NFR Framework [18] provides boundaries for the systematic representation of the logical dependencies and the tracking of non-functional requirements. It is an approach for modeling non-functional goals, comprising three different types of goals: soft goals, acting as quality attributes, satisficing goals, representing possibilities to fulfill a soft goal and argumentation goals, serving to record arguments regarding dependencies between the different elements.

To make quality assessment more tangible and pragmatic, most studies focus on the application of quality attributes in a particular domain or development phase. Consequently, quality attributes are often context-dependent [19]. Hence, in line with the objectives of our research, we now elaborate on evaluation concepts, mechanisms and tools.

\section{B. IS Evaluation and simulation quality}

Evaluation in IS deals with assessing the impact of an IS in terms of fulfilling intended purposes or delivering value. Quality attributes and concepts concerning IS evaluation mechanisms and systems mainly resemble general IS quality factors. Quality of Service (QoS) or service quality is a collective description for quality attributes in software service delivery and corresponding evaluation measures [16] and typically involves reliability, execution time and security [23]. Apart from formative and summative evaluation [1], several additional concepts exist to structure and classify evaluation approaches. Ex-ante evaluations focus on project feasibility and are performed before an investment is made [4], whereas ex-post evaluation assesses IT business value and examines the realization of expected IS benefits [3]. Moreover, evaluation of a system or artifact can be quantifiable (e.g. in terms of financial benefits) or qualitative (e.g. in terms of observed benefits based on opinions or judgement) [21]. Depending on the individual context, different evaluation mechanisms and models are available and appropriate [20]. However, no standards have yet been established, so that the choice of methods primarily depends on personal and organizational preferences [22]. In scientific literature and business practice, manifold evaluation frameworks for IS are available, however these would exceed the scope of this publication. A comprehensive overview about general IS evaluation can be found in [26], while particular artifact evaluation within DSR is elaborated on in [4].

Conducted and executed thoroughly as well as correctly, simulation modeling offers a reliable and useful way of computer-based system and artifact evaluation and provides valuable insights into expectable performance data of the IS [3]. Despite of the fact that computer simulation has become an eligible mechanism to evaluate IS (artifacts) [27], existing studies attempting to identify quality-related aspects of simulation modeling solely focus on a single simulation technique as well as the accuracy and credibility $[34,35,38]$ or the verification and validation of a model [30, 33] and neither provide a holistic overview about quality factors of simulation modeling nor a quality assessment framework for 
computer simulation in IS. According to the definition of [52], simulation assessment can be described as "a process by which interested parties (who were not involved in a model's origins, development and implementation) can determine, with some level of confidence, whether or not the model's results can be used in decision making". In contrast to general IS research, IS literature related to simulation does generally not refer to the general term of quality, but specifically elaborates on particular design elements such as individual model components or verification and validation procedures employed as quality assurance mechanisms [33]. A comprehensive overview about simulation-based research in the field of IS can be found in [25]. While simulation modelling has gained an increasing importance within the last decade, both in IS as well as interdisciplinary research, simulation models and studies are generally assessed in terms of their capability to deliver the intended outcomes, partially taking into account various influencing factors such as data quality or model fidelity [48]. Consequently, quality assurance for simulation modelling is commonly referred to as verification and validation, which in turn encompasses different methods and techniques (e.g. model validation by means of historical data, extreme condition tests, turing tests) depending on the given model choices [25]. Accordingly, several studies have proposed various guidelines and structural approaches for ensuring reliable development, verification and validation processes as well as outcomes to finally increase the credibility of simulation results $[30,33$, 34, 35]. The V-Model [36] for example is a software development process that demonstrates the relationships between different phases of the development life cycle and its associated phase of testing and was strongly influenced by the development-validation cycles proposed by simulation modelling theory [38]. However, while verification, validation, model fidelity and result credibility are commonly researched and considered in simulation modelling, a holistic quality view on simulation modelling in IS (as well as other research disciplines) has not been established yet. Ultimately, quality should encompass more elements than the credibility of results, which becomes particularly obvious when assessing large-scale simulation models that are practically impossible to verify and validate [30]. Consequently, quality criteria for simulation need to be developed to cover a wide range of additional factors such as the efficiency of simulation models when being executed [35] or the suitability of an existing model to be re-used in a new context [47]. Hence, we aim to close existing research gaps by extracting relevant quality factors and developing a framework for assessing the overall quality of a simulation.

\section{METhOdOLOGY AND RESEARCH DESIGN}

To address the specified research gaps, we have utilized a research design consisting of six stages. Thereby, we adhere to the principles of the Design Science Research Methodology (DSRM) [10] in IS and follow the objectives of this paper by means of an integrated DSRM cycle (Fig. 1).

From studying relevant IS literature, we have identified a lack of quality assurance in simulation modeling, especially within an IS evaluation context and consequently start with a problem-centered initiation. The objective of the first cycle was to develop a general framework for analyzing the quality of a computer simulation model, regardless of its individual purpose. For this reason, we propose a quality assessment framework for computer simulation based on the control method for simulation quality proposed by [51]. However, the design and development of the framework require a clear understanding of what constitutes to simulation quality. Hence, a second design cycle was initiated, in order to investigate individual, context-independent quality factors in simulation modeling. The aim of this cycle was to develop a comprehensive list of simulation quality factors as artifact. The factors were compiled in terms of a systematic literature review, which was conducted in line with the guidelines of [28]. To identify relevant literature concerning computer simulation modeling quality, we performed search queries in Google Scholar, AISeL and IEEE databases, which were selected in in line with their extensive scope of publications

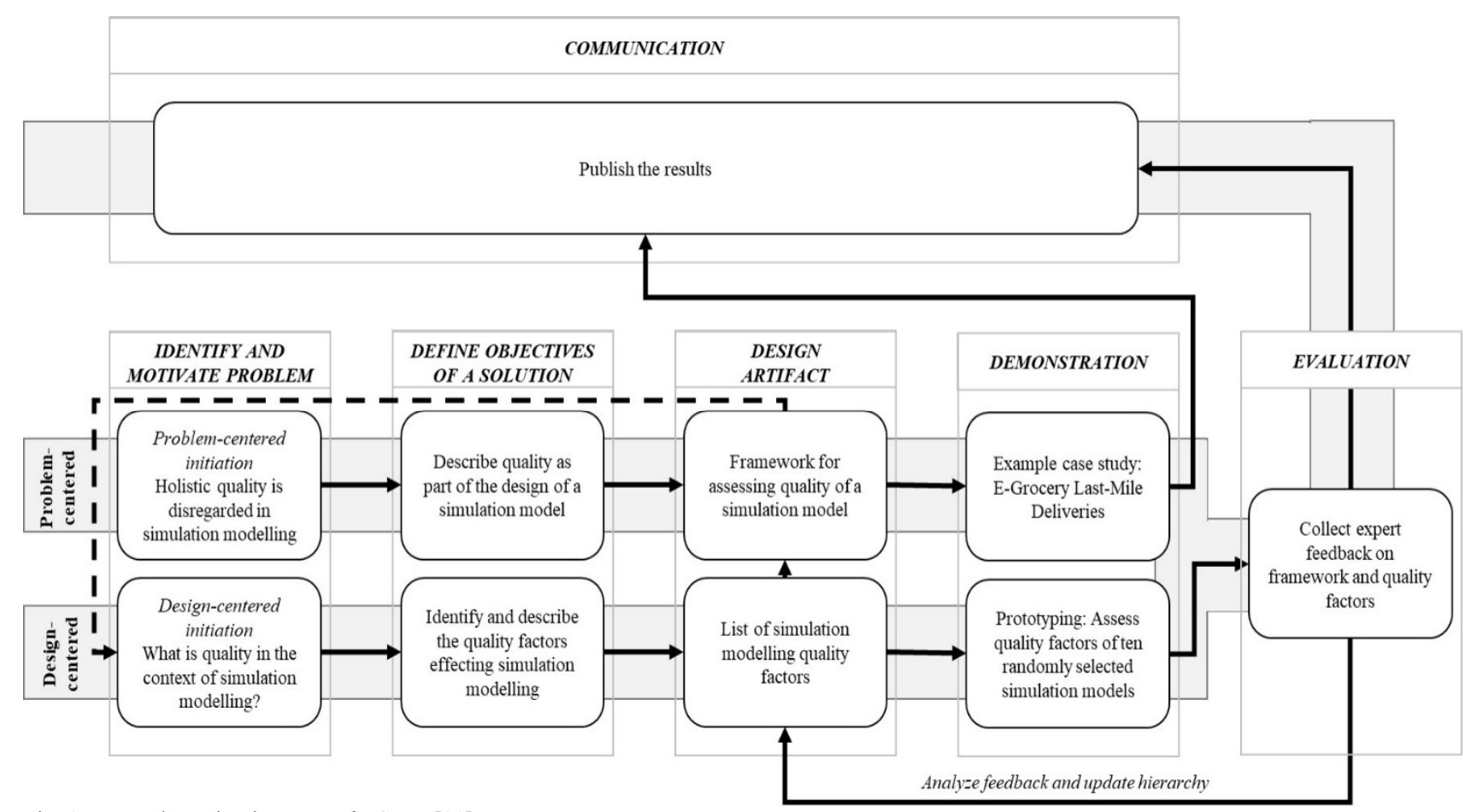

Fig. 1 Research Design in terms of DSRM [11] stages. 
TABLE I SYSTEMATIC LiteratURE SEARCH

\begin{tabular}{|c|c|c|c|c|}
\hline \multirow[b]{2}{*}{$\begin{array}{l}\text { Data- } \\
\text { base }\end{array}$} & \multicolumn{4}{|c|}{ Search framework } \\
\hline & Search Term & $\begin{array}{l}\text { Search } \\
\text { Fields }\end{array}$ & Hits & Relevant \\
\hline IEEE & \multirow{3}{*}{$\begin{array}{c}\text { "simulation" AND } \\
\text { ("model" OR } \\
\text { "modeling") AND } \\
\text { ("quality" OR "assess- } \\
\text { ment" OR "evaluation") }\end{array}$} & \multirow{3}{*}{$\begin{array}{l}\text { Title, } \\
\text { Abstract } \\
\text { and } \\
\text { Key- } \\
\text { words }\end{array}$} & 354 & 12 \\
\hline AISeL & & & 612 & 14 \\
\hline $\begin{array}{l}\text { Google } \\
\text { Scholar }\end{array}$ & & & 3.130 & 25 \\
\hline & & \multicolumn{2}{|c|}{ Backward search } & 4 \\
\hline & & \multicolumn{2}{|c|}{ Forward search } & 3 \\
\hline & & \multicolumn{2}{|c|}{ Related work } & 4 \\
\hline & & \multicolumn{2}{|r|}{ Total } & 62 \\
\hline
\end{tabular}

concerning simulation and IS research. An overview about the employed search terms, search fields and hits per database is given in Table 1. To exclude irrelevant sources and studies, we have implemented an inclusion criterion based on our research objectives. Consequently, only literature regarding quality in computer-based simulation approaches was reviewed, whereas other, non-computer-based simulation and modeling techniques were disregarded. Moreover, we excluded publications that were not published in journals related to the disciplines of information systems, management information systems, computer information systems, business information systems or simulation modelling. In the course of the literature review, we additionally assessed citations from the results of the literature search (backward approach), conducted a forward search by identifying the articles that quote important publications discerned during the search phase and integrated literature that has been considered for the related work section (Section II). Ultimately, we took into account 62 scientific sources (two books, four book chapters, 21 conference proceedings and 35 journal publications).

To account for contextual differences and acquire a conceptualization that can uniformly be utilized in an evaluation context, unclear or coinciding quality factor definitions were harmonized by means of an interpretivist stance. The compiled simulation quality factors were subsequently used as input for the quality assessment framework. To demonstrate the usefulness of the factors, we have applied them to a random selection of ten simulation models described in scientific literature. This form of prototyping provided valuable insights on the factors themselves as well as their properties. The revised quality factors were then updated in the assessment framework, which we included in an example case study regarding last-mile egrocery deliveries in Germany [29] to demonstrate its applicability. Demonstration is the first stop of evaluation in DSR and helps to assess particular elements of the design within the general research context. The final evaluation of the framework including our quality factors was conducted with the aid of expert feedback. Three independent experts with a work experience of $6-10$ years in the fields of simulation modeling and IS evaluation were interviewed in-depth on the framework as well as the quality factors. A qualitative analysis of the feedback provided additional guidance on adjusting and improving our design artifacts. As a result, several factors identified as inapplicable or futile for simulation quality assessment were removed, while a few additional factors proposed by the experts were introduced, reconciled or grouped to specific factors as their interpretations within the simulation quality context were highly coinciding. Finally, the varied feedback from the subject experts highlights the lack of common understanding of simulation quality, both in a general as well as an IS evaluation context.

\section{QUALITY FACTORS FOR SiMULATION MODELING}

In order to design, adapt or assess a simulation model for evaluating design artefacts at all stages of the IS development, a comprehensive overview of relevant quality factors as well as their individual impact and interdependencies is required. In the course of our literature review, we have identified 29 quality factors for simulation modeling, which we will present in the subsequent section. In accordance with IS quality literature, we have grouped the individual quality factors in line with the general quality concept of [31] and [51], establishing process, model/ content and result/ outcome as dimensions for simulation quality. This approach ensures a holistic focus on simulation quality at the process (modeling) stage as well as the implementation (model) stage and therefore allows prospective quality assurance and formative evaluation (ex-ante) as well as retrospective, summative quality assessment (ex-post). To demonstrate the adequacy of quality factors, their individual occurrence frequency in ten selected simulation studies is shown next to the particular abbreviation and outlined in section D.

\section{A. Quality of the process $(P)$}

The development of a reliable simulation model capable of producing meaningful as well as valid results requires quality assurance during the entire simulation modeling process [38]. The following factors aid in ensuring and evaluating the quality of a simulation model.

- Control $(P-C ; 6)$ : Control structures ensure a sound and objective development process and are intrinsic to the topic of investigation, consequently opposing a direct influence on the quality of the simulation model as well as decision making activities. Control measures can be regular routine meetings with the customer and/ or the project supervisor or timed feedback loops within the entire simulation team [32].

- Documentation (P-D; 5): Documentation material assists the user in assessing the use and functioning of a simulation model and therefore increases the model's transparency [33-37].

- $\quad$ Expertise (P-E; 6): The expertise and composition of a simulation team is crucial for ensuring a sound and productive modeling process. The expertise of a team or individual can be measured by the total amount of projects that have already been carried out as well as the amount of staff in a project with formal simulation training [38], [39]. Moreover, subjective measures such as the trustworthiness, honesty and commitment of the provider should be taken into account $[31,37]$.

- Involvement (P-I; 6): Involvement describes the degree of participation and cooperation of all parties involved in a simulation modeling project. It is a prerequisite for the transparency as well as usability and has a direct influence on the perceived completeness of a model [37, 39, 40].

- Relevance $(P-R ; 7)$ : This term is related to the object system as well as the modeling technique and the general technical development process. Simulation modelers need to ensure that they use an appropriate simulation language (specific or general-purpose) and 
simulation technique (discrete-event, agent-based, system dynamic) that is relevant to the given context, which will in turn also affect the conciseness of a model [34, 42]. Generally, large and complex models require object-oriented programming (OOP) technique as well as general-purpose simulation languages, while specific techniques and languages are more appropriate for small projects [41].

- $\quad$ Tool $(P-T ; 6)$ : The software used for the modeling process is a major influencing factor on the overall quality. A comprehensive tool needs to support object orientation and provide separate interfaces for users and developers [31, 37, 41, 43]. Moreover, interfaces for integrating external data sources should be supported and sophisticated visualization aids be given in order to foster the ease of validation.

- $\quad V \& V(P-V V ; 5):$ Verification and validation $(\mathrm{V} \& \mathrm{~V})$ are crucial to the overall quality of a simulation modeling process as well as the quality of the model and its outcomes. Hence, it needs to be ensured that a simulation model has been/ will be evaluated in-depth before (re-)employing it for an IS evaluation procedure. While verification ensures that a model is operating as it was intended, validation deals with assessing the reliability of its outcomes. The more intensive the $\mathrm{V} \& \mathrm{~V}$ processes, the higher the average quality can be expected to be $[34,41,44]$.

\section{B. Quality of the content $(C)$}

The content quality of simulation modeling refers to the simulation model itself and assesses quality characteristics of diverse model components.

- Accountability $(C-A C$; 6$)$ : The accountability of a simulation model refers to the potential ability of the model to measure its own usage. Simulation models featuring integrated probes or test mechanisms to measure timings or other relevant model elements can be assigned a higher quality status than models without any accountability characteristics [35].

- Applicability $(C-A P ; 10)$ : The purpose of a simulation model defines its fidelity and is vital for successful simulation projects. When formulating a problem for a simulation model, it should be ensured that it completely contains the actual problem, so that the simulation will be able to solve the right problem in a given setting $[38,45,46]$. In terms of quality, models that have been built without a specific purpose are inferior to problem-relevant (applicable) models.

- $\quad$ Augmentability (C-AU; 7): Simulation models should be set up in such a way that they can be expanded with regard to computational functions and/ or data storage specification to meet future requirements [35].

- $\quad$ Completeness (C-COM; 8): Completeness is directly related to the applicability of a simulation model. A simulation should represent all relevant aspects to solve the given problem and meet the demands of the user $[35,40]$. Hence, it describes the degree to which the simulation application captures all functional requirements and defines the model scope [33, 47].

- Conciseness (C-CONC; 7): High-quality simulation models are built with a minimum amount of code while still featuring execution efficiency in terms of execution times. Ideally, they are fragmented into sub-models, preventing the repeated use of identical code sequences, aiding in increased runtimes by eliminating false dependencies and unnecessary process serialization $[33,34,35,43]$.

- Consistency (C-CONS; 8): The formal quality of simulation models can also be evaluated by means of its structure. Uniformity concerning notations and terminology, definite structural patterns as well as a consistent use of attributes and variable types helps to understand the technical and conceptual functionalities of the simulation [35].

- Data quality (C-DQ; 10): Input data and model parameters have to be accurate, valid, complete and unbiased, regardless if they are left in their original form or if they have been transformed in the course of the modeling process [38, 39]. Inaccurate data directly impairs the accuracy of simulation results and consequently plays a superior role in simulation modeling [34, 37]. Data quality can be assured by means of the data quality assessment framework [11].

- Experimentability (C-E; 10): Experimentability describes the degree to which a simulation model offers capabilities for experimentation and includes parametrizability of input factors, pre-defined scenarios as well as optimization algorithms [41, 47].

- Fidelity $(C-F$; 10): A major driver of simulation quality is the quality or fidelity of the model utilized within the simulation. Depending on the applicability, a simulation model should accurately reflect the model's purpose within the real-system. Therefore, fidelity can also be described as degree to which a simulation model reproduces the state and behavior of a real-world object or system in an observable manner $[39,41]$. Mathematical measurements are suitable to determine fidelity by calculating the amount of identical elements shared between the simulation model and the real object or system [35].

- Interoperability (C-I; 3): Effective simulation models are capable of communicating and distributing data with other simulation applications to optimize the informational flow. Moreover, a model should be able to utilize information and data received from other applications for its own functionalities [33, 47].

- $\quad$ Maintainability (C-MA; 7): The term maintainability refers to the ease of maintaining and repairing a simulation model in case of malfunctions, defects, faulty components, unexpected working conditions or output errors and includes the model's dependability (degree to which the model delivers services as expected), availability (degree to which the model delivers services when requested) and reliability (degree to which the model works without failure). Furthermore, a simulation model with a high degree of maintainability is able to assign default values when run with no or invalid input variables and can consequently avoid some errors itself [33, 37, 38, 47].

- Measurability $(C-M E ; 10)$ : Measurability is the degree to which a model's fidelity can be assessed and compared to the real-system by means of measure 
instruments (e.g. visualizations) integrated into the simulation model [48].

- Parsimony (C-PA; 5): While conciseness refers to the code of a simulation model, parsimony is concerned with its content and therefore interrelated with a model's completeness. A good simulation model has to include key elements of the object/system and cover all perspectives relevant within the given context. However, at the same time, it should leave out gratuitous elements that do not serve to fulfill the model's purpose $[35,46]$.

- Portability (C-PO; 6): Basically, a simulation model consists of platform-specific and platformindependent elements. The more platformindependent elements are given, the easier it will be to run and refine the simulation model regardless of the available computer hardware configuration or technological changes [33, 35, 38, 47].

- Reusability $(C-R ; 8)$ : As mentioned before, existing models can be re-used to save time, efforts and costs. A model's capability to be re-used can be determined as reusability and is characterized by assessing in how far the model facilitates the selective use of its parts for other purposes (e.g. adapting the model or constructing a new model based on several of its fragments) $[31,37,47]$.

- Self-containedness (C-SC; 6): This quality factor evaluates the dependence of the simulation model on external parts, inputs, libraries or applications. A model performing all execution-relevant aspects internally is to be regarded superior as it does not rely on external sources, which can't be controlled in terms of availability and portability [35].

- Transparency $(C-T ; 8)$ : Components and functionnalities of a simulation model need to be transparent and both operating behavior as well as outputs should be easily understandable, so that observers can determine or verify the model's objectives, constraints, assumptions, components or results [35, 47]. Transparency is directly influenced by the documentation and the involvement of other parties and is a prerequisite for a model's usability [40].

- Usability (C-U; 6): Usability refers to the ease with which a simulation model can be utilized for its intended purpose. Both model as well as documentation should be easy to understand for (potential) users, consequently reducing the probability of simulation misuse. Therefore, user interfaces need to be designed in an appealing, selfdescribing way and input requirements, interaction possibilities (e.g. parametrization) as well as output forms and general software use should be neat and plain $[33,34,40,46]$.

- Visualizability $(C-V$; 7): Detailed animation and visualization properties can aid $V \& V$ processes by enabling the user to eyeball the behavior of the system and subsequently compare it to the real-world system $[40,48,50]$.

\section{Quality of the outcome $(O)$}

Despite of process and simulation model quality, one of the most important elements of simulation modeling is the analysis of results. In this context, result quality consists of two factors, namely accuracy and communicativeness.

- Accuracy $(O-A ; 10)$ : During the modeling process, models should be validated (ideally with real data) to ensure reliable and accurate results. Simulation outcomes that have been empirically tested and calibrated against real-world results promise a high overall degree of simulation quality. Outcome accuracy is directly related to the data quality and model fidelity. If no real data is available to calibrate

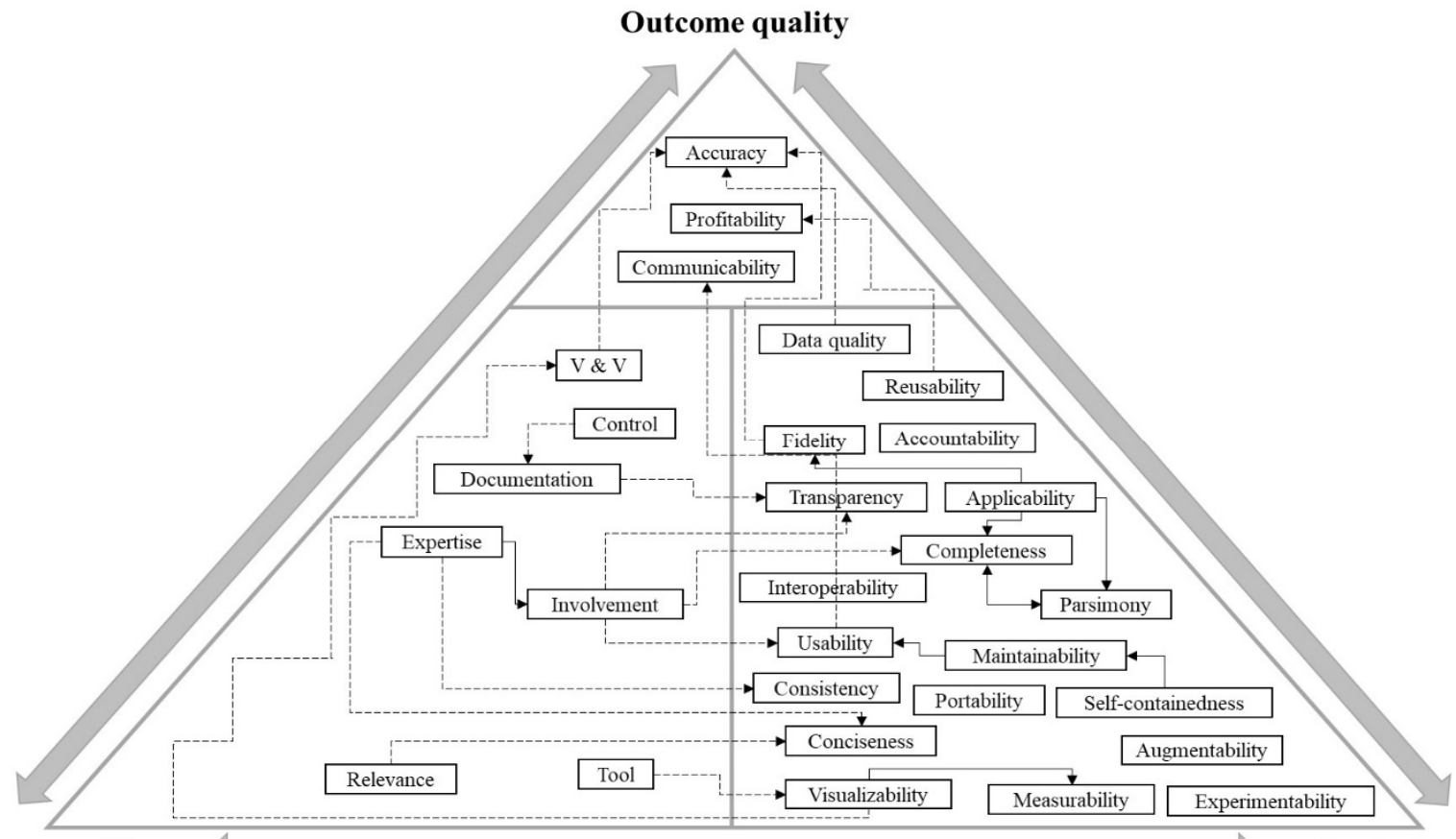

Process quality

Content quality

Fig. 2. Quality factor relationship across quality concept dimensions 
the model results, different strategies are available: (1) Adjust existing values to approximate desired variables, (2) construct values from other available records, (3) obtain estimates from experts, (4) utilize characteristic values from literature [33, 38, 39, 40].

- Communicability $(O-C ; 10)$ : The communicability of outcomes is related to the model's properties of providing outputs whose form and content are easy to assimilate and useful. Consequently, highly communicable results can be evaluated, processed and (re-)used in a more efficient manner, ultimately increasing the overall simulation quality [35, 42].

- Profitability $(O-P R ; 5)$ : Profitability can also be defined as economic efficiency and determines the cost-benefit constraint of a simulation model. It can be assessed by comparing all investments made for developing the simulation model to the overall savings/profits directly generated by the insights yielded from the simulation [34]. To influence the profitability, decision-makers can re-use existing models (short-term) or build up in-house simulation expertise (long-term) [49].

The delineated quality concepts and factors influence the overall simulation quality and are interrelated with each other to a limited extent. Direct or strong dependencies of quality factors are shown in Fig. 2. The direction of the arrows indicates the respective uni- (single arrow) or bidirectional (double arrow) dependency of the relationship. In this context, solid lines describe dependencies within the boundaries of a quality concept dimension, whereas dotted lines denote crossconcept interdependencies.

TABLE II. Quality Factors in Selected Simulation Models

\begin{tabular}{|c|c|c|c|}
\hline Process & Content & Outcome & Source \\
\hline $\begin{array}{c}P-E, P-R, \\
P-T\end{array}$ & $\begin{array}{c}C-A C, C-A P, C-C O M, C-C O N C, C-D Q, C- \\
E, C-F, C-M E, C-P A, C-P O, C-R, C-T, C- \\
V\end{array}$ & $\begin{array}{l}O-A, O- \\
C, O-P R\end{array}$ & [53] \\
\hline $\begin{array}{l}P-D, P-I, \\
P-R, P- \\
\quad V V\end{array}$ & $\begin{array}{c}C-A C, C-A P, C-A U, C-C O N C, C-C O N S, \\
C-D Q, C-E, C-F, C-I, C-M A, C-M E \\
C-P A, C-P O, C-R, C-S C, C-T, C-U\end{array}$ & $\begin{array}{l}O-A, O- \\
C\end{array}$ & [54] \\
\hline $\begin{array}{c}P-C, P- \\
D, P-R \\
P-T, P- \\
\quad V V \\
\end{array}$ & $\begin{array}{c}C-A P, C-C O M, C-C O N C, C-C O N S, \\
C-D Q, C-E, C-F, C-M A, C-M E, C-R, C- \\
S C, C-U, C-V\end{array}$ & $\begin{array}{l}O-A, O- \\
C, O-P R\end{array}$ & [55] \\
\hline $\begin{array}{l}P-C, P-E, \\
P-I, P-R, \\
P-T\end{array}$ & $\begin{array}{c}C-A P, C-A U, C-C O M, C-C O N C, C-D Q, C- \\
E, C-F, C-M A, C-M E, C-R, C-S C, \\
C-T, C-V\end{array}$ & $\begin{array}{l}O-A, O- \\
C\end{array}$ & [56] \\
\hline $\begin{array}{c}P-C, P- \\
D, P-E \\
P-I, P-R \\
P-T, P- \\
\quad V V\end{array}$ & $\begin{array}{c}C-A C, C-A P, C-A U, C-C O M, C-C O N C, C- \\
C O N S, C-D Q, C-E, C-F, C-I, C-M A, C- \\
M E, C-P O, C-R, C-T, C-U \\
C-V\end{array}$ & $\begin{array}{l}O-A, O- \\
C, O-P R\end{array}$ & [57] \\
\hline$P-R, P-T$ & $\begin{array}{c}C-A P, C-C O N S, C-D Q, C-E, C-F, C-M E, \\
C-P A, C-P O, C-U, C-V\end{array}$ & $\stackrel{O-A, O-}{C}$ & [58] \\
\hline$P-D, P-E$ & $\begin{array}{c}C-A C, C-A P, C-A U, C-C O M, C-C O N S, C- \\
D Q, C-E, C-F, C-M A, C-M E, C-R, \\
C-S C, C-T\end{array}$ & $\begin{array}{l}O-A, O- \\
C\end{array}$ & [59] \\
\hline $\begin{array}{l}P-C, P-I, \\
P-V V\end{array}$ & $\begin{array}{c}C-A P, C-A U, C-C O M, C-C O N C, C-C O N S \\
C-D Q, C-E, C-F, C-I, C-M A \\
C-M E, C-P A, C-P O, C-R, C-S C \\
C-T, C-U\end{array}$ & $\begin{array}{l}O-A, O- \\
C, O-P R\end{array}$ & [60] \\
\hline $\begin{array}{c}P-C, P- \\
D, P-E \\
P-I, P-R \\
P-T, P- \\
V V\end{array}$ & $\begin{array}{c}C-A C, C-A P, C-A U, C-C O M, C-C O N C, C- \\
C O N S, C-D Q, C-E, C-F, C-M E, C-P A, C-T \\
, C-U, C-V,\end{array}$ & $\begin{array}{l}O-A, O- \\
C, O-P R\end{array}$ & {$[61]$} \\
\hline $\begin{array}{l}P-C, P-E, \\
P-I\end{array}$ & $\begin{array}{c}C-A C, C-A P, C-A U, C-C O M, C-C O N S, C- \\
D Q, C-E, C-F, C-M A, C-M E, C-P O, C-R, \\
C-S C, C-T, C-V\end{array}$ & $\begin{array}{l}O-A, O- \\
C\end{array}$ & [62] \\
\hline
\end{tabular}

\section{Quality factors in simulation studies}

In order to demonstrate the use of our quality factors, we have measured their occurrence (or the occurrence of metrics related to these factors) in simulation models from ten randomly selected, successful simulation studies across various business disciplines. Therefore, we compiled a list of simulation studies published in scientific literature by employing the search term "simulation study" in the databases IEEE Xplore as well as Google Scholar and employed a random number generator to randomly draw ten studies for out demonstration process. Table 2 gives an overview about the individual occurrence of quality factors in these models. Each of the models assessed features at least five of our proposed quality factors. Considering all models, every quality factor occurs at least five times (whereby the factors applicability, data quality, experimentability, fidelity, measurability, accuracy and communicability occur in every study), which likely demonstrates the appropriateness und usefulness of our quality factors. Due to a lack of information, we did not assign quality factors that were not denoted in a paper, even though the factor may have actually been taken into account during the study.

\section{A FrAMEWORK For ASSESSING THE QUALITY OF SIMULATION MODELS}

In this section, based on the proposed quality factors, we present a quality assessment framework for simulation modeling in order to support the quality of simulation models employed for the evaluation of IS. The assessment framework is an expansion of the simulation quality control method [51] shown in Fig. 3. The method equates simulation quality with simulation credibility and establishes V\&V processes concerning both data as well as the simulation as prerequisites of quality assurance during the entire simulation life cycle. $\mathrm{V} \& \mathrm{~V}$ activities support the quality of the simulation in terms of data, model, process and results. Subsequently, these elements will be assessed in terms of their credibility, whereby credibility is defined as "measurement of the degree that simulation meets the application purpose, which is determined by similarity between simulation system and real-system" [51]. While a simulation model always presents an abstraction of the reality and consequently the real-system, simulation credibility refers to the level of confidence of the user or decision maker that the simulation is capable of mimicking all relevant system states of the real-system in a reliable manner. As soon as the credibility meets the purpose to a satisfactory extent, which may require several modification iterations, the simulation can be accepted. While the simulation quality control method offers a valuable basis for simulation assessment, it lacks a holistic view on quality. By equating

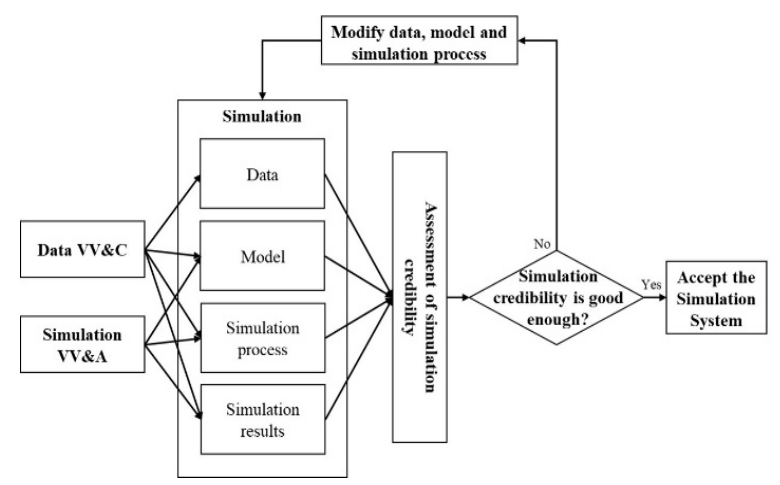

Fig. 3 Simulation quality control method [59] 
quality with credibility, essential aspects potentially influencing the overall quality of a simulation are disregarded and could lead to bad-decision making or wrong evaluation insights into IS. For instance, a simulation might be regarded as credible if the model and its results are directly reflected by insights from the real system. However, this does not ensure a high, all-embracing simulation modeling quality, as it might have taken years and huge investments to ensure the simulation credibility, so that other evaluation instruments would have been more appropriate or useful. Another example can be given in the case of portability: When considering to employ an existing (credible) simulation model to evaluate IS artifacts, despite of its credibility, the model might turn out to be invalid or inappropriate for the new context due to a lack of portability. Therefore, omitting important decision factors such as profitability or portability can impair the assessment of simulation modeling or models as evaluation tool for IS.

Consequently, we have adapted the basic simulation quality control method by integrating our proposed quality factors to feature a holistic and reliable view on simulation quality. The details of the adapted model are shown in Fig 4. The content concentric factor data quality as well as the process concentric factor $V \& V$ have a direct influence on the quality of results and therefore act as prerequisites for the general simulation approach. Hence, both in ex-ante as well as ex-post evaluation, these factors should be ensured or analyzed first. Subsequently, the simulation should be assessed in terms of its process quality factors (Section IV-A), content quality factors (Section IV-B) and outcome quality factors (Section IV-C) to indemnify quality across all dimensions of simulation modeling. Thereby, the individual weighting of quality factors is subjective and depends on context and purpose of the simulation. By iteratively reviewing the quality factors, relevant modifications and substantiated decision on the use of simulation as IS evaluation tool can be made.

\section{A. Examplary Case Study: E-Grocery}

To demonstrate the usefulness of the quality assessment framework for simulation, we have employed it in an exemplary case. The framework is instantiated by means of a conceptual model, used to assess the quality of a simulation model that has been built to benchmark the ecological impacts of grocery deliveries on the last mile in an urban context [29]. The partner organization for this case is looking to quantify different emission outputs resulting from e-grocery as well as

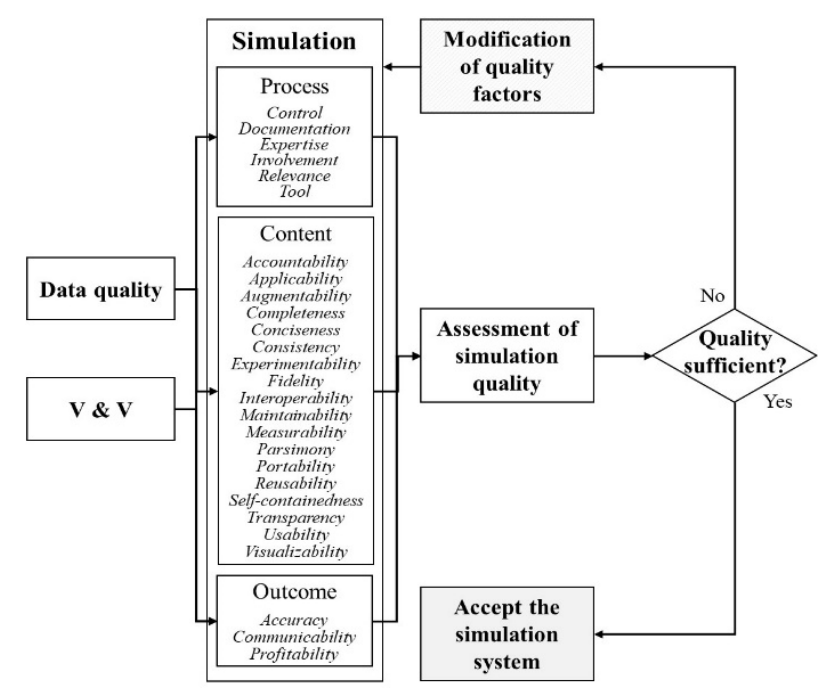

Fig. 4 Simulation quality assessment framework for IS evaluation stationary grocery shopping in a selected study area, in order to allow for a sound comparison and evaluation of grocery shopping concepts in terms of their environmental value and to identify constraints (e.g. maximum tour distances, amount of delivery vehicles) required for increasing the ecologic value of deliveries initiated by online grocery shopping. Therefore, simulation model insights and results are employed to evaluate the information provided by the company's internal information systems and to generate the respective outputs for the objectives stated above. Emissions are deduced from the individual mileage of delivery and private vehicles and converted into $\mathrm{CO}, \mathrm{CO}_{2}, \mathrm{~N}_{2} \mathrm{O}, \mathrm{NH}_{3}$ and $\mathrm{NO}_{\mathrm{x}}$ outputs.

In the first step, we assessed both data quality as well as the presence and rigorous application of V\&V procedures. As the data was either gathered from relevant literature sources or directly from the partner organization and V\&V procedures were in place during the entire simulation development project, the two pre-qualifying factors can be regarded as sufficient to move on with the quality assessment procedure. Consequently, we started to assess the quality of the simulation development process, model and results by employing the quality factors presented in Section IV. Despite of a lack of simulation expertise within the development team, the process has proven to satisfy relevant quality criteria: (1) Both model and process were documented in detail, (2) control structures were used during the development, (3) the degree of involvement between provider and partner organization were high and (4) simulation tool as well as techniques used for the modeling process seemed appropriate and useful within the given context. Concerning the content, the model featured and served a clear purpose (applicability), consistent structure (consistency), integrated experiments (experimentability) and a high degree of fidelity, which was assessed by obtaining expert feedback from the partner organization. Moreover, additional quality factors such as measurability (by comparing fictive and real tour data), reusability (the model is built in such a way that it can represent a huge variety of scenarios concerning product deliveries) and self-containedness (all databases and inputs are stored within the model itself) are fulfilled. However, as the simulation has been built in a specific simulation tool, the degree of portability is restricted as the tool itself is required to run and modify the model. Here, the quality could be increased by converting the simulation model into an executable application (e.g. JAVA) in order to provide a device- and platform-independent runtime environment and hence increase the portability. In terms of outcome-specific quality, the results of the simulation have been confirmed and validated by real data (see measurability) and can easily be communicated (emission values are easy to understand and of major relevance to many decision-makers). Accordingly, accuracy and communicability can be regarded as achieved.

After having assessed all quality factors, we have found the simulation quality sufficient for the given purpose, which was also confirmed by the partner organization and consequently demonstrates the effectiveness of our simulation assessment framework. Even though not all quality factors were met (e.g. expertise and portability), the overall quality was still sufficient for all parties involved, indicating the importance of context as well as purpose for the role and weighting of individual quality factors. 


\section{CONCLUSION}

This paper outlines a variety of quality factors relevant for the design and assessment of simulation models as evaluation instruments for IS, which is a contemporary trend, as simulation modeling has become an effective and popular tool for evaluating complex and large systems. To date, simulation quality has mainly been described in terms of accuracy or credibility, which, however, lacks the necessary holistic view on quality. To address this research gap, we have proposed a set of quality factors that has been elicited and reviewed by demonstrating its usefulness by means of prototyping as well as collecting expert feedback. The quality factors are described in detail and their potential role in the evaluation of simulation quality is illustrated by employing them in a sophisticated simulation quality assessment framework. We adapted a given simulation quality control method to incorporate the proposed quality factors to enable a systematic evaluation approach, whereby the eligibility of the framework is proven by employing it in an example case.

The particular focus of this contribution has been the quality regarding simulation modeling and simulation models. Both quality factors as well as framework allow the integrated or separated assessment of the simulation process, model and results. While our study was designed to establish quality factors for simulation as evaluation instrument in IS, the factors can also be used for general quality assurance in simulation modeling. Therefore, our contribution has several practical implications. First of all, it can aid in increasing the overall quality of IS design by ensuring appropriate and efficient simulation evaluation mechanisms. Moreover, it supports simulation-related decision-making processes by establishing clear and relevant guidelines for the creation of new simulation models as well as the assessment of existing models. Both the proposed set of quality factors as well as the quality assessment framework can aid in establishing organizational quality assurance programs and models, ultimately contributing to the design of new business models as well as the overall quality of IS [64]. However, while our quality factors and framework can uniformly be employed across different domains and industries, their individual peculiarity and weighting depends on the individual context. Consequently, they form the basis for devising methodological guidelines for the design and development of simulation models and require relation to and revision in different business contexts in future research.

\section{SUPPLEMENTARY MATERIAL}

Bibliographical information on the literature reviewed in the course of our literature review can be found under DOI: 10.21227/a2fa-km47

\section{REFERENCES}

[1] S. Gregor and A. R. Hevner, "Positioning and Presenting Design Science Research for Maximum Impact," MIS Quarterly, vol. 37, no. 2, pp. 337-355, 2013.

[2] A. R. Hevner, S. T. March, J. Park, and S. Ram, "Design Science in Information Systems Research,” MIS Quarterly, vol. 28, no. 1, p. 75, 2004 .

[3] K. Kumar, "Post implementation evaluation of computer-based information systems: current practices," Commun. ACM, vol. 33 , no. 2 , pp. 203-212, 1990.

[4] N. Prat, I. Comyn-Wattiau, and J. Akoka, "A Taxonomy of Evaluation Methods for Information Systems Artifacts," Journal of Management Information Systems, vol. 32, no. 3, pp. 229-267, 2015.
[5] R. A. Wurbs, "Reservoir System Simulation and Optimization Models," Journal of Water Resources Planning and Management, vol. 119, no. 4, pp. 455-472, 1993.

[6] H. Roßnagel, J. Zibuschka, and O. Junker, "Agent-Based Simulation for Evaluation of a Mobile Emergency Management System," in Lecture Notes in Business Information Processing, vol. 58, Sustainable e-Business Management: AMCIS 2010, M. L. Nelson, Ed., Berlin, Heidelberg: Springer-Verlag Berlin Heidelberg, 2010, pp. 100-114.

[7] H. Takeda, P. Veerkamp, and H. Yoshikawa, "Modeling Design Process", AIMag, vol. 11, no. 4, pp. 37-48, 1990.

[8] O. I. Lindland, G. Sindre, and A. Solvberg, "Understanding quality in conceptual modeling," IEEE Softw., vol. 11, no. 2, pp. 42-49, 1994.

[9] T. Saarinen, "An expanded instrument for evaluating information system success," Information \& Management, vol. 31, no. 2, pp. 103118, 1996.

[10] K. Peffers, T. Tuunanen, M. A. Rothenberger, and S. Chatterjee, "A Design Science Research Methodology for Information Systems Research," Journal of Management Information Systems, vol. 24, no. 3, pp. 45-77, 2007.

[11] D. L. Moody and G. G. Shanks, "Improving the quality of data models: empirical validation of a quality management framework," Information Systems, vol. 28, no. 6, pp. 619-650, 2003.

[12] L. L. Pipino, Y. W. Lee, and R. Y. Wang, "Data quality assessment," Commun. ACM, vol. 45, no. 4, p. 211, 2002.

[13] R. Y. Wang and D. M. Strong, "Beyond Accuracy: What Data Quality Means to Data Consumers," Journal of Management Information Systems, vol. 12, no. 4, pp. 5-33, 1996.

[14] E. W. Duggan and H. Reichgelt, Eds., Measuring information systems delivery quality. Hershey, Pa: IGI Global (701 E. Chocolate Avenue Hershey Pennsylvania 17033 USA), 2006.

[15] D. E. Perry, N. A. Staudenmayer, and L. G. Votta, "People, organizations, and process improvement," IEEE Softw., vol. 11, no. 4, pp. 36-45, 1994.

[16] L. F. Pitt, R. T. Watson, and C. B. Kavan, "Service Quality: A Measure of Information Systems Effectiveness," MIS Quarterly, vol. 19, no. 2, p. $173,1995$.

[17] H. Reijers and J. Mendling, "Modularity in Process Models: Review and Effects," in Business process management: 6th international conference, BPM 2008, Milan, Italy, September 2 - 4, 2008 ; proceedings, Berlin, Heidelberg, 2008, pp. 20-35.

[18] L. Chung, B. A. Nixon, E. Yu, and J. Mylopoulos, "The NFR Framework in Action," in International Series in Software Engineering, vol. 5, Non-Functional Requirements in Software Engineering, L. Chung, B. A. Nixon, E. Yu, and J. Mylopoulos, Eds., Boston, MA: Springer, 2000, pp. 15-45.

[19] C. Batini, C. Cappiello, C. Francalanci, and A. Maurino, "Methodologies for data quality assessment and improvement," $A C M$ Comput. Surv., vol. 41, no. 3, pp. 1-52, 2009.

[20] G. Marthandan and C. M. Tang, "Information systems evaluation: an ongoing measure," IJBIS, vol. 6, no. 3, p. 336, 2010.

[21] J. Ward, E. Daniel, and J. Peppard, "Building Better Business Cases for IT Investments," MIS Quarterly Executive, vol. 7, no. 1, pp. 1-15, 2008.

[22] K. M. Rosacker and D. L. Olson, "An Empirical Assessment of IT Project Selection and Evaluation Methods in State Government," Project Management Journal, vol. 39, no. 1, pp. 49-58, 2008.

[23] K. Kritikos et al., "A survey on service quality description," $A C M$ Comput. Surv., vol. 46, no. 1, pp. 1-58, 2013.

[24] B. A. Kitchenham and L. Jones, "Evaluating software engineering methods and tools part 6," SIGSOFT Softw. Eng. Notes, vol. 22, no. 2, pp. 16-18, 1997.

[25] J. Beese, M. K. Haki, S. Aier, and R. Winter, "Simulation-Based Research in Information Systems," Bus Inf Syst Eng, vol. 61, no. 4, pp. 503-521, 2019, doi: 10.1007/s12599-018-0529-1.

[26] Z. Irani, "Information systems evaluation: navigating through the problem domain," Information \& Management, vol. 40, no. 1, pp. 1124, 2002.

[27] P. J. MacArthur, R. L. Crosslin, and J. R. Warren, "A strategy for evaluating alternative information system designs for business process reengineering," International Journal of Information Management, vol. 14, no. 4, pp. 237-251, 1994. 
[28] J. Webster, and R.T. Watson, "Analyzing the past to prepare for the future: writing a literature review," MIS Quarterly, 26(2), 2002, pp. xiii-xxiii.

[29] M. Auf der Landwehr, M. Trott, and C. von Viebahn, "E-Grocery in Terms of Sustainability - Simulating the Environmental Impact of Grocery Shopping for an Urban Area in Hanover," in Simulation in Produktion und Logistik, M. Putz, and A. Schlegel, Eds., Auerbach, Verlag Wissenschaftliche Scripten, 2019, pp. 87-96.

[30] R. G. Sargent et al., "Strategic directions in verification, validation, and accreditation research," in 2000 Winter Simulation Conference, Orlando, FL, USA, Dec. 2000, pp. 909-916.

[31] S. Robinson, "General concepts of quality for discrete-event simulation," European Journal of Operational Research, vol. 138, no. 1, pp. 103-117, 2002, doi: 10.1016/S0377-2217(01)00127-8.

[32] V. Hlupic and G. J. de Vreede, "Business process modelling using discrete-event simulation: current opportunities and future challenges," IJSPM, vol. 1, 1/2, p. 72, 2005, doi: 10.1504/IJSPM.2005.007115.

[33] O. Balci, "Quality Assessment, Verification, and Validation of Modeling and Simulation Applications," in 2004 Winter Simulation Conference, Washington, D.C, 2004, pp. 116-123.

[34] T. I. Ören, "Concepts and criteria to assess acceptability of simulation studies: a frame of reference," Commun. ACM, vol. 24, no. 4, pp. 180189, 1981, doi: 10.1145/358598.358605.

[35] O. Balci, "How to assess the acceptability and credibility of simulation results," in Proceedings of the 21st conference on Winter simulation, Washington, D.C., United States, 1989, pp. 62-71.

[36] J. O. Clark, "System of Systems Engineering and Family of Systems Engineering from a standards, V-Model, and Dual-V Model perspective," in 2009 3rd Annual IEEE Systems Conference, Vancouver, BC, Canada, Mar. 2009 - Mar. 2009, pp. 381-387.

[37] S. Robinson, "Measuring service quality in the process of delivering a simulation study: The customer's perspective," International Transactions in Operational Research, vol. 5, no. 5, pp. 357-374, 1998, doi: 10.1016/S0969-6016(98)00025-2.

[38] O. Balci, "Guidelines for successful simulation studies," in Proceedings of Winter Simulation Conference, New Orleans, LA, USA, 1990, pp. 25-32.

[39] S. Robinson and M. Pidd, "Provider and customer expectations of successful simulation projects," Journal of the Operational Research Society, vol. 49, no. 3, pp. 200-209, 1998, doi: 10.1057 /palgrave.jors.2600516.

[40] D. J. van der Zee and J. G. A. J. van der Vorst, "A Modeling Framework for Supply Chain Simulation: Opportunities for Improved Decision Making*," Decision Sciences, vol. 36, no. 1, pp. 65-95, 2005, doi: 10.1111/j.1540-5915.2005.00066.x.

[41] T. I. Ören, M. S. Elzas, and G. Sheng, "Model reliability and software quality assurance in simulation of nuclear fuel waste management systems," SIGSIM Simul. Dig., vol. 16, no. 4, pp. 4-19, 1985, doi: 10.1145/1102581.1102582.

[42] J. G. A. J. van der Vorst, S.-O. Tromp, and D.-J. van der Zee, "Simulation modelling for food supply chain redesign; integrated decision making on product quality, sustainability and logistics," International Journal of Production Research, vol. 47, no. 23, pp. 6611-6631, 2009, doi: 10.1080/00207540802356747.

[43] P. McNally and C. Heavey, "Developing simulation as a desktop resource," International Journal of Computer Integrated Manufacturing, vol. 17, no. 5, pp. 435-450, 2004, doi: $10.1080 / 09511920310001654283$.

[44] O. Balci, "Principles and techniques of simulation validation, verification, and testing," in 1995 Winter Simulation Conference: Proceedings, December 3-6, 1995, Arlington, VA, USA, 1995, pp. 147-154.

[45] R. G. Sargent, "Verifying and validating simulation models," in Winter Simulation Conference (WSC), 2014: 7-10 Dec. 2014, Savannah, GA, Savanah, GA, USA, 2014, pp. 118-131.

[46] R. G. Sargent, "An introductory tutorial on verification and validation of simulation models," in Proceedings of the 2015 Winter Simulation Conference, December 6-9, 2015, Huntington Beach, CA, Huntington Beach, CA, USA, 2015, pp. 1729-1740.

[47] A. B. AL-Badareen, M. H. Selamat, M. A. Jabar, J. Din, and S. Turaev, "Software Quality Models: A Comparative Study," in Communications in Computer and Information Science Ser, Software Engineering and
Computer Systems, Part I: Second International Conference, ICSECS 2011, Kuantan, Malaysia, June 27-29, 2011, pp. 46-55.

[48] D. Liu, N. Macchiarella, and D. Vincenzi, "Simulation Fidelity," in EBL-Schweitzer, Human factors in simulation and training, P. Hancock, D. Vincenzi, J. Wise, M. Mouloua, and P. A. Hancock, Eds., Hoboken: CRC Press, 2008, pp. 61-73.

[49] J. Becker, M. Rosemann, and C. von Uthmann, "Guidelines of Business Process Modeling," in Lecture Notes in Computer Science, vol. 1806, Business process management: Models, techniques, and empirical studies, W. van der Aalst, Ed., Berlin [etc.]: Springer, 2000, pp. 30-49.

[50] B. Rohrmann, S. Palmer, and I. Bishop, "Perceived quality of computer-simulated environments," in Environment-behavior research on the Pacific Rim: Proceedings paper, G. Moore, J. Hunt, and L. Trevillon, Eds., Sydney, New South Wales, Faculty of Architecture: University of Sydney, 2000, pp. 341-352

[51] Y. Tan, J. Wang, and W. Zhang, "Discussion on credibility theory of simulation application," in System Simulation and Scientific Computing, 2008. ICSC 2008. Asia Simulation Conference - 7th International Conference on, Beijing, China, 2008, pp. 368-374.

[52] S. I. Gass, "Feature Article-Decision-Aiding Models: Validation, Assessment, and Related Issues for Policy Analysis," Operations Research, vol. 31, pp. 603-631, 1983, doi: 10.1287/opre.31.4.603.

[53] N. Agatz, A. L. Erera, M. W.P. Savelsbergh, and X. Wang, "Dynamic Ride-Sharing: a Simulation Study in Metro Atlanta," Procedia - Social and Behavioral Sciences, vol. 17, pp. 532-550, 2011, doi: 10.1016/j.sbspro.2011.04.530.

[54] W. van Heeswijk, R. Larsen, and A. Larsen, "An urban consolidation center in the city of Copenhagen: A simulation study," International Journal of Sustainable Transportation, vol. 13, no. 9, pp. 675-691, 2019, doi: 10.1080/15568318.2018.1503380.

[55] D. Ivanov, "Revealing interfaces of supply chain resilience and sustainability: a simulation study," International Journal of Production Research, vol. 56, no. 10, pp. 3507-3523, 2018, doi: 10.1080/00207543.2017.1343507.

[56] Y. Ren, H. Guo, G. Liu, and H. Ye, "Simulation Study of Geometric Characteristics and Coverage for Moon-Based Earth Observation in the Electro-Optical Region," IEEE J. Sel. Top. Appl. Earth Observations Remote Sensing, vol. 10, no. 6, pp. 2431-2440, 2017, doi: 10.1109/JSTARS.2017.2711061.

[57] L. Schwab, S. Gold, and G. Reiner, "Exploring financial sustainability of SMEs during periods of production growth: A simulation study," International Journal of Production Economics, vol. 212, pp. 8-18, 2019, doi: 10.1016/j.ijpe.2018.12.023.

[58] J. Bischoff, M. Maciejewski, and K. Nagel, "City-wide shared taxis: A simulation study in Berlin," in IEEE ITSC 2017: 20th International Conference on Intelligent Transportation Systems: Mielparque Yokohama in Yokohama, Kanagawa, Japan, October 16-19, 2017, Yokohama, 2017, pp. 275-280.

[59] A. H. Gharehgozli, F. G. Vernooij, and N. Zaerpour, "A simulation study of the performance of twin automated stacking cranes at a seaport container terminal," European Journal of Operational Research, vol. 261, no. 1, pp. 108-128, 2017, doi: 10.1016/j.ejor.2017.01.037.

[60] V. Albino, L. Fraccascia, and I. Giannoccaro, "Exploring the role of contracts to support the emergence of self-organized industrial symbiosis networks: an agent-based simulation study," Journal of Cleaner Production, vol. 112, pp. 4353-4366, 2016, doi: 10.1016/j.jclepro.2015.06.070.

[61] K. Calvi, F. Halawa, M. Economou, R. Kulkarni, and S. H. Chung, "Simulation study integrated with activity-based costing for an electronic device re-manufacturing system," Int J Adv Manuf Technol, vol. 103, 1-4, pp. 127-140, 2019, doi: 10.1007/s00170-019-03429-3.

[62] C. Archetti, M. G. Speranza, and D. Weyland, "A simulation study of an on-demand transportation system," International Transactions in Operational Research, vol. 25, no. 4, pp. 1137-1161, 2018, doi: 10.1111/itor.12476.

[63] T. K. Abdel-Hamid, "The Economics of Software Quality Assurance: A Simulation-Based Case Study," MIS Quarterly, vol. 12, no. 3, p. 395, 1988, doi: $10.2307 / 249206$

[64] R. Knackstedt, S. Bräuer, and T. Schoormann, "Tool Support for Designing Innovative Sustainable Business Models." in The art of structuring, K. Bergener, M. Räckers, A. Stein, Eds., Schweiz: Springer, 2019, pp 87-100. 